\title{
Wavelet Entropy Based Algorithm for Fault Detection and Classification in FACTS Compensated Transmission Line
}

\author{
Amany M. El-Zonkoly, Hussein Desouki \\ Department of Electric \& Control Engineering, Collage of Engineering \& Technology, \\ Arab Academy for Science \& Technology, Alexandria, Egypt \\ E-mail: amanyelz@yahoo.com \\ Received November 3, 2010; revised December 10, 2010; accepted December 15, 2010
}

\begin{abstract}
Distance protection of transmission lines including advanced flexible AC transmission system (FACTS) devices has been a very challenging task. FACTS devices of interest in this paper are static synchronous series compensators (SSSC) and unified power flow controller (UPFC). In this paper, a new algorithm is proposed to detect and classify the fault and identify the fault position in a transmission line with respect to a FACTS device placed in the midpoint of the transmission line. Discrete wavelet transformation and wavelet entropy calculations are used to analyze during fault current and voltage signals of the compensated transmission line. The proposed algorithm is very simple and accurate in fault detection and classification. A variety of fault cases and simulation results are introduced to show the effectiveness of such algorithm.
\end{abstract}

Keywords: FACTS, SSSC, UPFC, Wavelet Transform, Entropy Calculation

\section{Introduction}

In recent years, it has become more difficult to construct new generation facilities and transmission lines due to energy and environmental problems. Hence, it is required to enhance the power transfer capability of existing transmission lines instead of constructing new ones. Because of all that, it became more important to control the power flow along the transmission lines to meet the needs of power transfer. On the other hand, FACTS devices have received more attention in transmission system operations as they can be utilized to alter power system parameters in order to control power flow. With FACTS technology, such as static var compensators (SVCs), static synchronous compensators (STATCOMs), static synchronous series compensators (SSSCs) and unified power flow controllers (UPFCs), etc., bus voltages, line impedances and phase angles in the power system can be flexibly and rapidly regulated. In addition, the FACTS devices have the capability of increasing transmission capabilities, decrease the generation cost and improve the security and stability of power system $[1,2]$. During fault, the presence of compensating devices affects steady-state and transient components of current and voltage signals which create problems with relay functionality $[3,4]$.
Fault classification and section identification in a transmission line with FACTS devices is a very challenging task. Some researchers used current and voltage signals to determine the fault location and fault resistance only without attempting to find the fault type and phase involved [5]. Earlier an adaptive Kalman filtering approach has been proposed for protection of uncompensated power distribution networks [6] and compensated transmission system employing an advanced series compensator [7]. However, the Kalman filtering approach finds its limitation, as fault resistance cannot be modeled and further it requires a number of different filters to accomplish the task. Different types of neural networks (NN) based pattern recognition procedures [7-9] were proposed which large training need set generation, large training time and design of a new neural network for each transmission line. Different attempts have been made for fault location and classification using numerical methods, wavelet transform, S-transform, TT-transform, fuzzy logic systems and support vector machines [3-15]. Most of these attempts were trying to classify the fault and identify the faulted section in a transmission line compensated either by series capacitor protected by metal-oxide varistor (MOV) or compensated by thyristor-controlled series compensators (TCSCs) protected by MOV or compensated by both. 
In [5], authors took advantage of the post-fault voltage and current samples taken synchronously from both ends of the line to build a recursive optimization algorithm to find the distance to fault in a transmission line compensated with a series FACTS device. The proposed algorithm in [5] is independent of the FACTS device model. However, it aimed only to the location of fault without trying to find its type.

In this paper, we are interested in two of the most important FACTS devices; the SSSC and the UPFC. The SSSCs are FACTS devices for power transmission line series compensation. It is a power electronic-based voltage source converter (VSC) that generates a nearly sinusoidal three-phase voltage which is in quadrature with the line current. The SSSC converter block is connected in series with the transmission line by series coupling transformer. The SSSC can provide either capacitive or inductive series compensation independent of the line current [16]. The UPFC, which has been recognized as one of the best featured FACTS devices, is capable of providing simultaneous active and reactive power flow control, as well as, voltage magnitude control. The UPFC is a combination of STATCOM and SSSC which are connected via a common DC link, to allow bidirectional flow of real power between series output terminals of SSSC and the shunt terminals of the STATCOM [2]. These two devices are suggested due to some problems encountered in case of lines compensated with conventional compensators such as fixed series capacitor or TCSC. Problems encountered in case of series compensated lines are as follows [12]:

1) The steady state current is increased significantly with series compensation and it may be greater than the line-to-ground fault current towards the boundary of the line.

2) In a typical series compensation arrangement, the metal oxide varistor (MOV) is used to protect the capacitor from over-voltages during a fault. However, it acts non-linearly during faults and increases the complexity of the protection problem.

3) Voltage and current inversions.

4) The voltage and current signals produced on the transmission line contain different frequency components such as non fundamental decaying as well as decaying DC components due to resonance between the system inductance and series capacitor, odd harmonics due to MOV conduction during faults, sub-synchronous frequencies having frequency components varying around half the fundamental frequency value, high frequency components caused by resonance between line capacitance and line inductance and fundamental components of the steady state fault current.

The proposed algorithm is more general it uses voltage and current signals recorded at one end of the line with no need for synchronization and is independent of modes of operation of FACTS devices. The proposed algorithm is simple and applied to both symmetrical and unsymmetrical faults with no need for pre-trained NN.

For the purpose of fault identification and classification, the wavelet entropy theory is applied to produce a simple and accurate algorithm. Wavelet transform (WT) has good time-frequency localization ability so it particularly adapted to analyze the singular signals caused by fault. Wavelet transform provides theory basis for fault detection. The most effective method for fault detection is using a universal applicable quantity (UAQ) to describe the system and detect the fault. Shannon entropy is such a UAQ, and wavelet entropy (WE) is formed by combining WT and Shannon entropy together [17]. A combination of wavelet and entropy, could exploit the advantages of both methods to describe the characteristics of a signal. This is because wavelet meets the demands of transient signal analysis and entropy is ideal for the measurement of uncertainty.

In [18], the proposed algorithm was applied to a non-compensated transmission line. Therefore, current waveforms only are used. In this paper due to the presence of FACTS devices the steady-state and transient components of current and voltage signals are much affected which create problems with fault detection, classification and phase selection. The faulted phase couldn't be determined using current waveforms coefficients only. For this reason, the three phase voltages waveforms are also needed to determine the phase included in fault in case of SLG fault after the compensating device. That is why the proposed algorithm in this paper, although it is simple, it is more detailed and complicated than that introduced in [18].

In this paper, a test system is built using SIMULINK. The resulting data under different fault types and position with respect to the compensating device are analyzed using the modified WE algorithm than that in [18] to consider the system compensation. The test results show the effectiveness of the proposed algorithm.

\section{Wavelet Transform and Entropy Calculations}

Lots of fault information is included in the transient components. So it can be used to identify the fault or abnormity of equipments or power system. It can also be used to deal with the fault and analyze its reason. This way the reliability of the power system will be considerably improved.

Transient signals have some characteristics such as high frequency and instant break. Wavelet transform is 
capable of revealing aspects of data that other signal analysis techniques miss and it satisfies the analysis need of electric transient signals. Usually, wavelet transform of transient signal is expressed by multi-revolution decomposition fast algorithm which utilizes the orthogonal wavelet bases to decompose the signal to components under different scales. It is equal to recursively filtering the signal with a high-pass and low-pass filter pair. The approximations are the high-scale, low-frequency components of the signal produced by filtering the signal by a low-pass filter. The details are the low-scale, highfrequency components of the signal produced by filtering the signal by a high-pass filter. The band width of these two filters is equal. After each level of decomposition, the sampling frequency is reduced by half. Then recursively decompose the low-pass filter outputs (approximations) to produce the components of the next stage $[19,20]$.

Given a discrete signal $\mathrm{x}(\mathrm{n})$, being fast transformed at instant $\mathrm{k}$ and scale $\mathrm{j}$, it has a high-frequency component coefficient $\mathrm{D}_{\mathrm{j}}(\mathrm{k})$ and a low-frequency component coefficient $A_{j}(k)$. The frequency band of the information contained in signal components $D_{j}(k)$ and $A_{j}(k)$, obtained by reconstruction are as follows [21].

$$
\left\{\begin{array}{l}
D_{j}(k):\left[2^{-(j+1)} f_{s}, 2^{-j} f_{s}\right] \\
A_{j}(k):\left[0,2^{-(j+1)} f_{s}\right]
\end{array}(j=1,2, \cdots, m)\right.
$$

Where, $f_{s}$ is the sampling frequency.

The original signal sequence $\mathrm{x}(\mathrm{n})$ can be represented by the sum of all components as follows [21].

$$
\begin{aligned}
x(n) & =D_{1}(n)+A_{1}(n)=D_{1}(n)+D_{2}(n)+A_{2}(n) \\
& =\sum_{j=1}^{J} D_{j}(n)+A_{J}(n)
\end{aligned}
$$

Various wavelet entropy measures were defined in [19]. In this paper, the nonnormalized Shannon entropy will be used. The definition of nonnormalized Shannon entropy is as follows [21].

$$
E_{j}=-\sum_{k} E_{j k} \log E_{j k}
$$

Where $E_{j k}$ is the wavelet energy spectrum at scale $j$ and instant $\mathrm{k}$ and it is defined as follows.

$$
E_{j k}=\left|D_{j}(k)\right|^{2}
$$

\section{Proposed Algorithm for Transmission Line Fault Detection and Identification}

During fault, the amplitude and frequency of the test signal will change significantly as the system change from normal state to fault. The Shannon entropy will change accordingly. It becomes incapable of dealing with some abnormal signals while wavelet can. Wavelet combined entropy can make full use of localized feature at time-frequency domains. Wavelet analysis deals with unsteady signal while information entropy expresses information of the signal. That is why wavelet entropy can analyze fault signals more efficiently $[17,19,20]$.

The proposed algorithm detects if there is a fault or the compensated system is under normal conditions. It also determines the position of the fault if it is after or before the compensating device. In addition, the algorithm determines the type of fault if it is a single line to ground (SLG) fault, line to line (L-L) fault, double line to ground (DLG) fault or a three line to ground (3LG) fault. Finally, the algorithm selects the phases involved in the fault.

The transient signals of the three phase currents and voltages are produced using the simulation model built with the power block set of the SIMULINK. A discrete wavelet transformation is performed using two level symmetric wavelet for the three phase current signals $\left(i_{a}\right.$, $i_{b}$ and $i_{c}$ ) and the ground current ig, where

$$
i_{g}=i_{a}+i_{b}+i_{c}
$$

The entropy of each coefficient of the four currents is then calculated. The sum of absolute entropies of such coefficients for each current is then calculated (suma, sumb, sumc and sumg). The sums related to the three phase currents are then arranged to determine the maximum sum $(\max 1)$ the minimum sum $(\min 1)$ and the intermidiate sum $(\max 2)$.

The wavelet and entropy calculation are performed also for the three phase voltages in case the algorithm detected a single line to ground fault after the compensating device. The entropy sums of the three phase voltages are used to determine which phase is included in the fault.

The proposed algorithm is applied in three main steps. First, the fault is detected then its type and position with respect to the compensating device are determined. Finally, the phases included in the fault are identified. A detailed flow chart of the proposed algorithm is shown in Figure 1 which proceeds as follows:

- If sumg $<$ th 1 a No Fault condition is declared.

- If sumg $>$ th1 and sumg $<1$ then check on max 1 If $\max 1<$ th 2 a No Fault condition is declared Else if $\max 1>$ th 2 then it is a LL Fault. Further check max 1 to determine the fault position with respect to the FACTS device where,

If $\max 1<$ th 3 then the fault is after the FACTS device

Else the fault is before. 


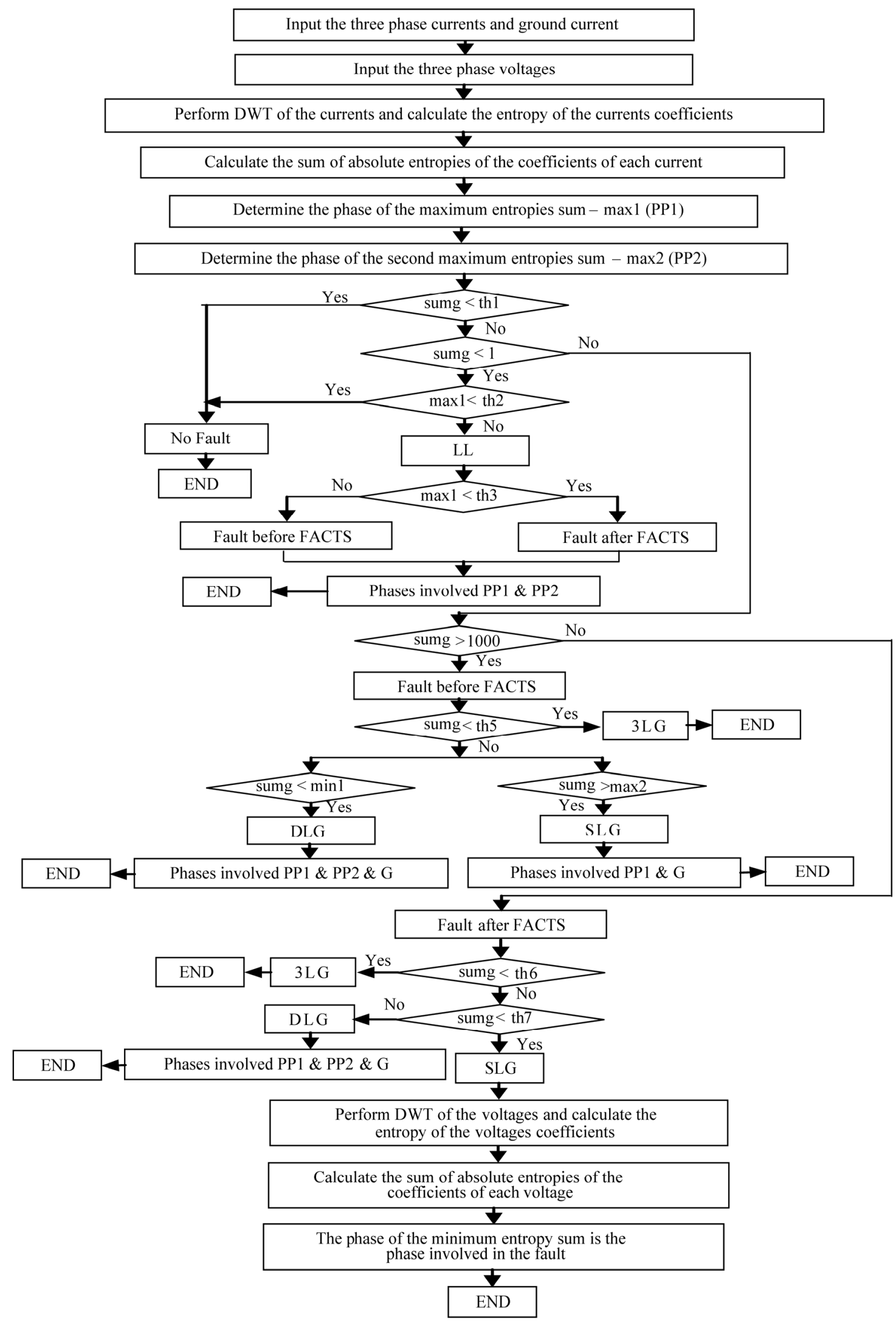

Figure 1. Flow chart of the proposed algorithm. 
- If sumg $>$ th1 but sumg $>1$ then check sumg again where,

If sumg $>1000$ then the fault is before the FACTS device

Else the fault is after.

- To determine the fault type whether it is after or before the FACTS device proceed as in the following steps.

- For a fault before the FACTS device,

If sumg $<$ th 5 then it is a 3 LG Fault

Else if sumg $>$ th5 then check

if sumg $>\max 2$ then it is a SLG Fault

else if sumg $<\min 1$ then it is a DLG Fault.

- For a fault after the FACTS device,

If sumg $<$ th 6 then it is a 3 LG Fault

Else if sumg $>$ th 6 then check

If $\max 1>$ th7 then it is a DLG Fault

Else it is a SLG Fault.

- Finally, after determining the location and type of each fault, the phases involved in each fault is determined as follows,

- for a LL fault the phases involved in the fault will be PP1 and PP2.

- for a DLG fault the phases involved in the fault will be PP1 and PP2 in addition to ground.

- for a SLG fault before the FACTS device the phase involved in the fault will be PP1.

- for a SLG fault after the FACTS device the selection of the phase included in fault was not possible using sum of currents entropies. Therefore, the sum of entropies of the coefficients of each of the phase voltages were calculated and the phase with the minimum sum was considered as the faulted phase.

\section{Test System}

Using the power system blockset (PSB) and the SIMULINK software, the test system is simulated. The test system is shown in Figure 2 and its data are listed in the Appendix.

\section{Simulation Results}

As mentioned before the test system was compensated by two different FACTS devices, SSSC and UPFC. In the following the simulation results of the system with the SSSC are given first then the results with the UPFC are given next. The simulation frequency was $10 \mathrm{kHz}$.

\subsection{System Compensated with SSSC}

For different fault types before and after the SSSC the

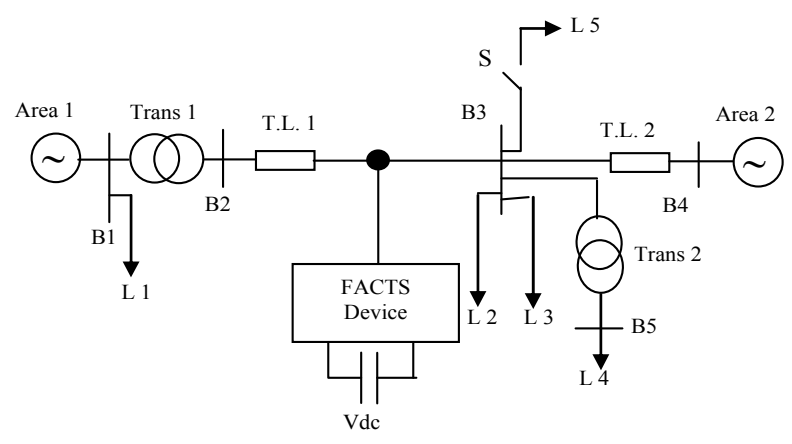

Figure 2. Power system model.

sum of absolute entropies of the coefficients of each current is given in Table $\mathbf{1}$.

As shown in Table 1, in case of no fault or in case of connecting extra load (L5) to the system, sumg was less than th1which is equal to $1 \times 10^{-8}$ for faults either before or after the FACTS device. It was also noticed that in case of SLG fault after the SSSC the selection of the phase included in fault was not possible using sum of currents entropies as it is in case of fault before the SSSC. For example, for an AG fault before the SSSC, suma is greater than sumb and sumc. However, for an AG fault after the SSSC, suma is greater than sumb but not sumc. Therefore, the sum of entropies of the coefficients of each of the phase voltages were calculated and the phase with the minimum sum was considered as the faulted phase. The sum of entropies of the coefficients of the phase voltages in case of a SLG fault after SSSC are given in Table 2. As shown in Table 2, for an AG fault after the SSSC, suma is less than sumb and sumc.

As a sample, the waveforms of the three phase currents in case of 3 LG fault before the SSSC are shown in Figure 3. The wavelet coefficients (approximate A2, level 1 detail D1 and level 2 detail D2) of phase A current are shown in Figure 4. In the same way, the waveforms of the three phase currents in case of $3 \mathrm{LG}$ fault after the SSSC and the wavelet coefficients of phase A current are shown in Figure 5 and Figure 6.

\subsection{System Compensated with UPFC}

For different fault types before and after the UPFC the sum of absolute entropies of the coefficients of each current is given in Table 3 .

As shown in Table 3, in case that sumg was greater than th1which is equal to $1 \times 10^{-8}$, greater than 1 , but less than 1000 , there will be a fault located after the FACTS device. It was also noticed that in case of SLG fault after the UPFC the selection of the phase included in fault was not possible using sum of currents entropies as it is in case of fault before the UPFC. For example, for a BG 
Table 1. The sum of absolute entropies of the coefficients of each current before and after SSSC.

\begin{tabular}{|c|c|c|c|c|c|c|c|c|}
\hline \multirow{2}{*}{ Fault Type } & \multicolumn{4}{|c|}{ Before $\times 10^{6}$} & \multicolumn{4}{|c|}{ After $\times 10^{6}$} \\
\hline & suma & sumb & sumc & sumg & suma & sumb & sumc & Sumg $\times 10^{-6}$ \\
\hline $\mathrm{AG}$ & 1.48 & 1.18 & 1.06 & 2.09 & 1.05 & 0.94 & 1.15 & 26.2 \\
\hline BG & 0.98 & 1.34 & 1.24 & 1.75 & 1.04 & 1.03 & 0.99 & 22.34 \\
\hline $\mathrm{CG}$ & 1.15 & 1.02 & 1.45 & 1.88 & 0.89 & 1.08 & 1.09 & 24.57 \\
\hline $\mathrm{AB}$ & 5.5 & 4.86 & 0.99 & $0.045 \times 10^{-6}$ & 2.29 & 1.94 & 0.93 & 0.17 \\
\hline $\mathrm{BC}$ & 0.91 & 3.6 & 2.96 & $0.043 \times 10^{-6}$ & 0.88 & 1.88 & 1.54 & 0.15 \\
\hline $\mathrm{CA}$ & 5.27 & 0.94 & 6.04 & $0.0517 \times 10^{-6}$ & 2.01 & 0.85 & 2.48 & 0.14 \\
\hline $\mathrm{ABG}$ & 5.91 & 4.61 & 1.06 & 0.59 & 2.24 & 1.84 & 0.89 & 20.73 \\
\hline BCG & 0.98 & 3.77 & 2.99 & 0.74 & 0.86 & 1.72 & 1.43 & 21.77 \\
\hline $\mathrm{CAG}$ & 5.39 & 1.01 & 6.03 & 0.60 & 1.95 & 0.78 & 2.33 & 21.67 \\
\hline $3 \mathrm{LG}$ & 8.20 & 4.64 & 5.3 & 0.33 & 2.93 & 3.17 & 2.18 & 9.78 \\
\hline Loading & 0.99 & 1.02 & 1.08 & 0 & 0.99 & 1.03 & 1.08 & 0.3 \\
\hline No Fault & 0.98 & 1.02 & 1.07 & 0 & 0.98 & 1.02 & 1.07 & 0 \\
\hline
\end{tabular}

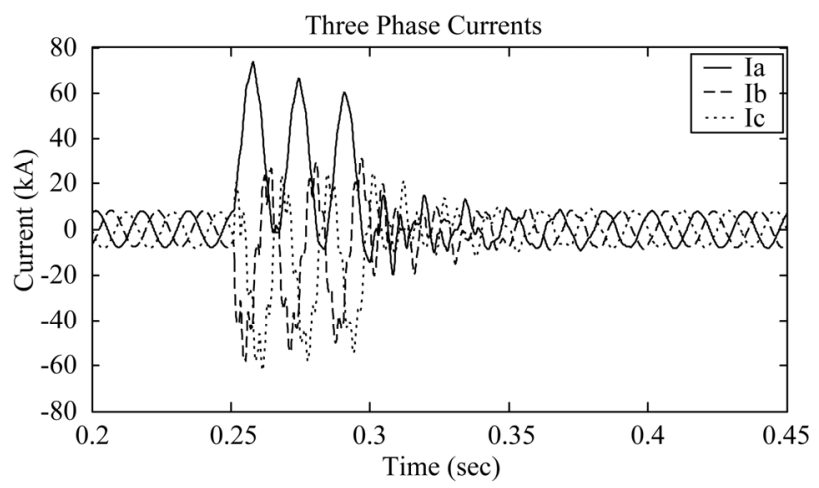

Figure 3. Three phase current waveforms during 3LG fault before the SSSC.
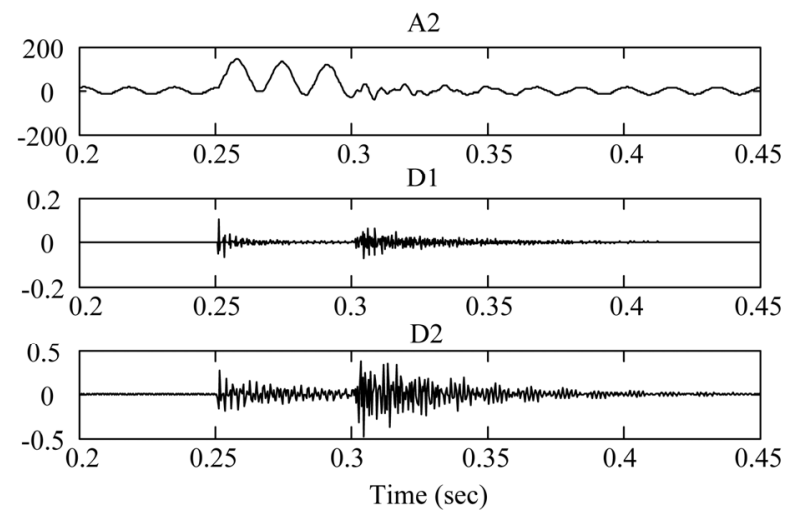

Figure 4. Approx. and details of phase A current during 3LG fault before SSSC.

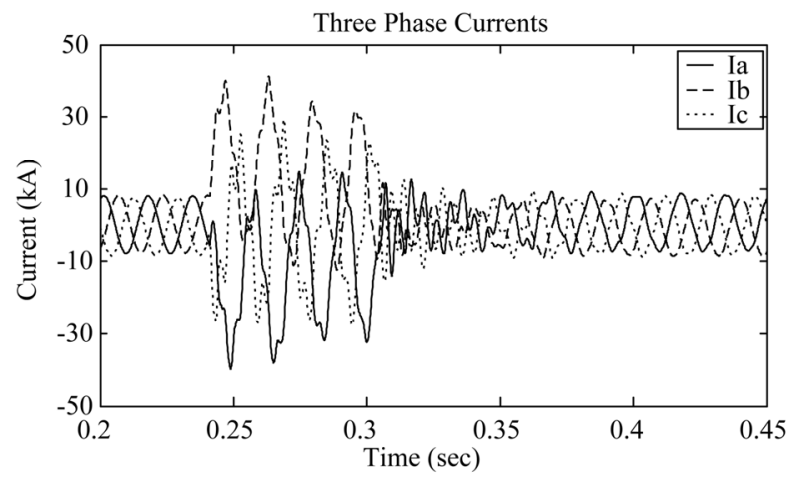

Figure 5. Three phase current waveforms during 3LG fault after the SSSC.
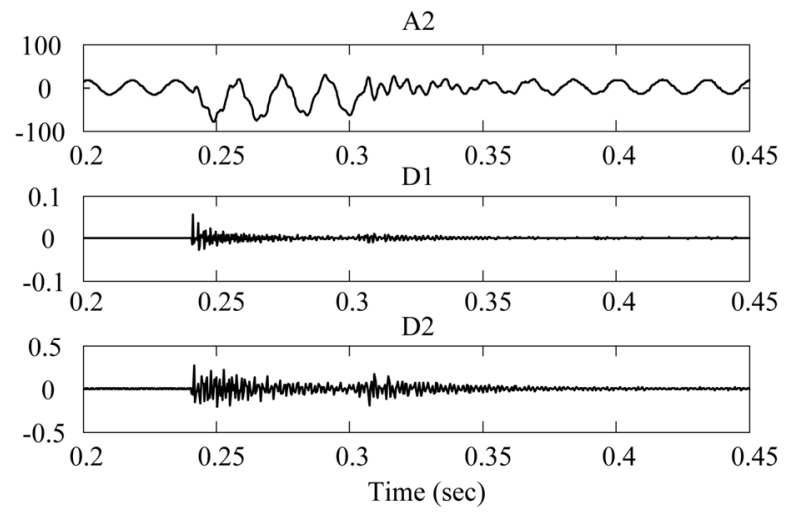

Figure 6. Approx. and details of phase A current during 3LG fault after SSSC. 
Table 2. The sum of entropies of the coefficients of the phase voltages in case of a SLG fault after SSSC.

\begin{tabular}{cccc}
\hline Fault Type & sum a & sum b & sum c \\
\hline AG & $3.4885 \times 10^{3}$ & $3.5568 \times 10^{3}$ & $3.5539 \times 10^{3}$ \\
BG & $3.5476 \times 10^{3}$ & $3.5149 \times 10^{3}$ & $3.5551 \times 10^{3}$ \\
CG & $3.5418 \times 10^{3}$ & $3.5631 \times 10^{3}$ & $3.5022 \times 10^{3}$ \\
\hline
\end{tabular}

fault before the UPFC, sumb is greater than suma and sumc. However, for an BG fault after the UPFC, sumb is greater than sumc but not suma. For this reason, as in case of SSSC compensation, the phases included in a SLG fault after the UPFC were determined using the voltage entropies. The sum of entropies of the coefficients of each of the phase voltages were calculated and the phase with the minimum sum was considered as the faulted phase. The sum of entropies of the coefficients of the phase voltages in case of a SLG fault after UPFC are given in Table 4. As shown in Table 4, for a BG fault after the UPFC, sumb is less than suma and sumc.

As a sample, the waveforms of the three phase currents in case of $3 \mathrm{LG}$ fault before the UPFC are shown in Figure 7. The wavelet coefficients (approximate A2, level 1 detail D1 and level 2 detail D2) of phase A current are shown in Figure 8. In the same way, the wave forms of the three phase currents in case of $3 L G$ fault after the UPFC and the wavelet coefficients of phase A current are shown in Figure 9 and Figure 10.

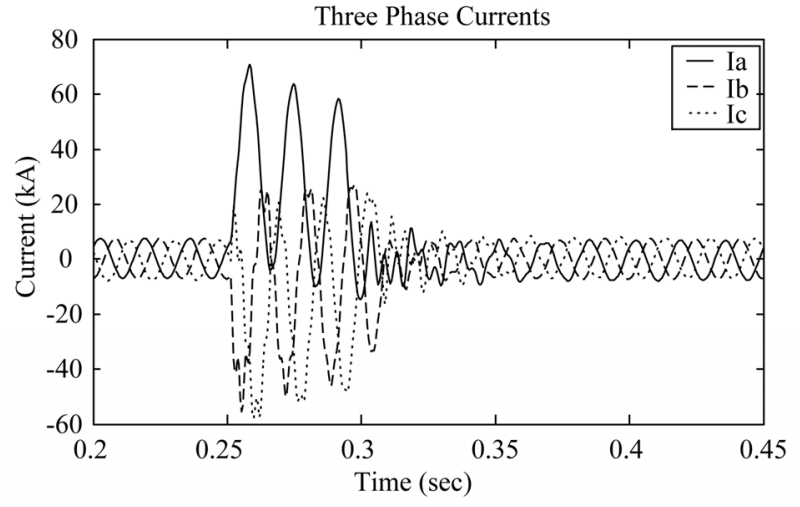

Figure 7. Three phase current waveforms during 3LG fault before the UPFC.
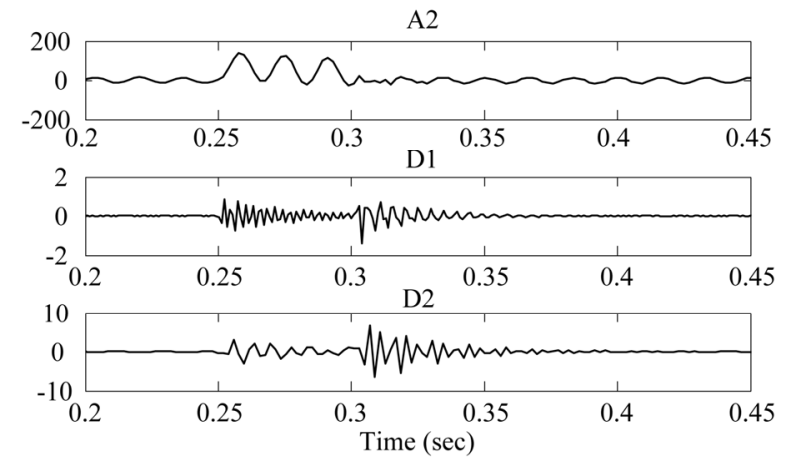

Figure 8. Approx. and details of phase A current during 3LG fault before UPFC.

Table 3. The sum of absolute entropies of the coefficients of each current before and after UPFC.

\begin{tabular}{ccccccccc}
\hline \multirow{2}{*}{ Fault Type } & \multicolumn{3}{c}{ Before $\times 10^{5}$} & & \multicolumn{3}{c}{ After $\times 10^{5}$} \\
\cline { 2 - 6 } & suma & sumb & sumc & sumg & suma & sumb & sumc & sumg $\times 10^{-5}$ \\
\hline AG & 2.82 & 1.92 & 1.83 & 3.59 & 1.95 & 1.52 & 2.09 & 5.069 \\
BG & 1.59 & 2.52 & 2.23 & 2.84 & 1.79 & 1.789 & 1.76 & 4.054 \\
CG & 1.98 & 1.58 & 2.93 & 3.13 & 1.51 & 1.81 & 2.11 & 4.592 \\
AB & 9.98 & 8.57 & 1.82 & $0.0746 \times 10^{-5}$ & 4.29 & 3.59 & 1.75 & 0.0024 \\
BC & 1.56 & 6.66 & 5.39 & $0.0321 \times 10^{-5}$ & 1.51 & 3.49 & 3.042 & 0.0002 \\
CA & 9.83 & 1.56 & 11.44 & $0.0773 \times 10^{-5}$ & 3.98 & 1.48 & 4.92 & 0.0749 \\
ABG & 10.7 & 8.16 & 1.87 & 0.918 & 4.31 & 3.49 & 1.78 & 3.548 \\
BCG & 1.61 & 6.98 & 5.52 & 1.19 & 1.56 & 3.42 & 3.05 & 4.913 \\
CAG & 10.1 & 1.62 & 11.51 & 0.9564 & 4.015 & 1.52 & 4.79 & 3.762 \\
3LG & 15.1 & 8.7 & 10.35 & 0.289 & 5.535 & 3.75 & 4.504 & 2.288 \\
Loading & 1.69 & 1.69 & 1.95 & $0.047 \times 10^{-5}$ & 1.69 & 1.69 & 1.95 & 0.047 \\
No Fault & 1.66 & 1.66 & 1.92 & 0 & 1.66 & 1.66 & 1.92 & 0 \\
\hline
\end{tabular}


Table 4. The sum of entropies of the coefficients of the phase voltages in case of a SLG fault after UPFC.

\begin{tabular}{cccc}
\hline Fault Type & sum a & sum b & sum c \\
\hline AG & 652.423 & 673.6073 & 673.517 \\
BG & 666.0345 & 662.1051 & 674.0677 \\
CG & 663.4631 & 674.5866 & 661.2325 \\
\hline
\end{tabular}

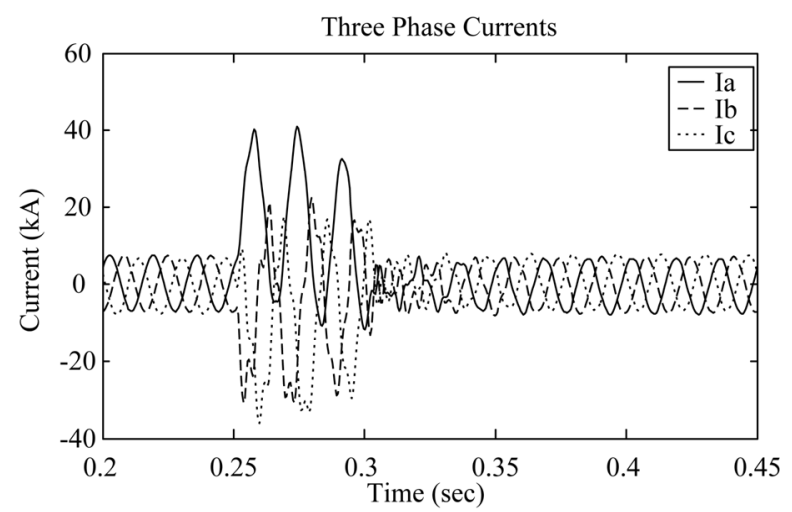

Figure 9. Three phase current waveforms during 3 LG fault after the UPFC.
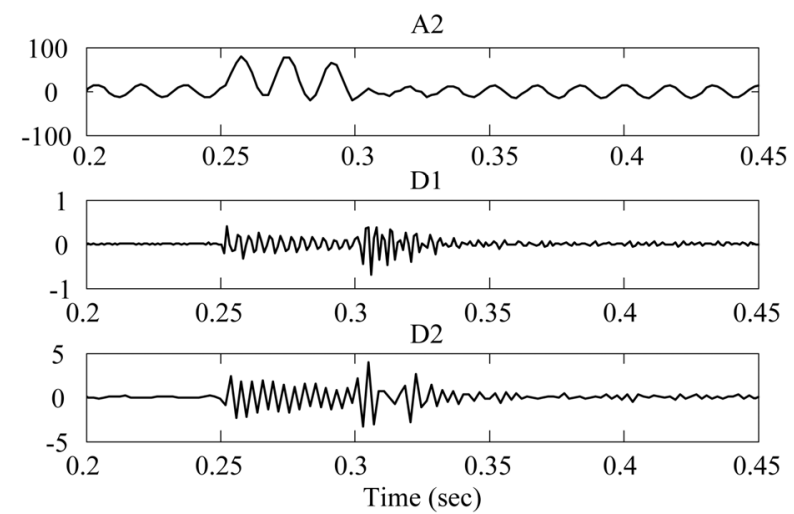

Figure 10. Approx. and details of phase A current during 3LG fault after UPFC.

\section{Conclusion}

As shown in the paper, the proposed algorithm was very accurate and simple in the same time. The algorithm succeeded in detecting the fault, determining its type and position with respect to compensating device and identifying the phases included in fault. Test results showed the effectiveness of the proposed algorithm under any type and position of fault.

\section{References}

[1] E. Uzunovic, "EMTP Transient Stability and Power Flow
Models and Controls of VSC Based FACTS Controllers," Ph.D. Dissertation, University of Waterloo, Waterloo, 2001.

[2] B. Geethalakshmi, and P. Dananjayan, "Investigation of Performance of UPFC without DC Link Capacitor," International Journal of Electric Power Systems Research, Vol. 78, No. 4, April 2007, pp. 736-746. doi:10.1016/j. epsr.2007.05.019

[3] P. K. Dash and S. R. Samantray, "Phase Selection and Fault Section Identification in Thyristor Controlled Series Compensated Line Using Discrete Wavelet Transform," International Journal of Electrical Power \& Energy Systems, Vol. 26, No. 9, September 2004, pp. 725-732. doi: 10.1016/j.ijepes.2004.05.005

[4] A. I. Megahed, A. Monem Moussa and A.E.Bayoumy, "Usage of Wavelet Transform in the Protection of Series-Compensated Transmission Lines," IEEE Transactions on Power Delivery, Vol. 21, No. 3, July 2006, pp. 1213-1221. doi:10.1109/TPWRD.2006.876981

[5] J. Sadeh and A. Adinehzadeh, "Accurate Fault Location Algorithm for Transmission Line in the Presence of Series Connected FACTS Devices," International Journal of Electrical Power \& Energy Systems, Vol. 32, No. 4, May 2010, pp.323-328. doi:10.1016/j.ijepes.2009.09.001

[6] S. R. Samantaray, P. K. Dash and S. K. Upadhyay, "Adaptive Kalman Filter and Neural Network Based High Impedance Fault Detection in Power Distribution Networks," International Journal of Electrical Power \& Energy Systems, Vol. 31, No. 4, May 2009, pp. 167-172. doi:10.1016/j.ijepes.2009.01.001

[7] S. R. Samantray and P. K. Dash, "Pattern Recognition Based Digital Relaying for Advanced Series Compensated Line," International Journal of Electrical Power \& Energy Systems, Vol. 30, No. 1, February 2008, pp. 102 112. doi:10.1016/j.ijepes.2007.06.018

[8] S. Suja and J. Jerome, "Pattern Recognition of Power Signal Disturbances Using S Transform and TT Transform," International Journal of Electrical Power \& Energy System, Vol. 32, No. 1, January 2010, pp. 37-53. doi: 10.1016/j.ijepes.2009.06.012

[9] Z. He, S. Gao, X. Chen, J. Zhang, Z. Bo and Q. Qian, "Study of a New Method for Power System Transients Classification Based on Wavelet Entropy and Neural Network," International Journal of Electrical Power \& Energy Systems, Article in Press, 2010. doi:10.1016/j. ijepes.2010.10.001

[10] P. S. Bhowmik, P. Purkait and K. Bhattacharya," A Novel Wavelet Transform Aided Neural Network Based Transmission Line Fault Analysis Method," International Journal of Electrical Power \& Energy Systems, Vol. 31, No. 5, June 2009, pp. 213-219

[11] A. A. Eisa and K. Ramar, "Accurate One-End Fault Location for Overhead Transmission Lines in Interconnected Power Systems," International Journal of Electrical Power \& Energy Systems, Vol. 32, No. 5, June 2010, pp. 383-389. doi:10.1016/j.ijepes.2009.11.005

[12] U. B. Parikh, B. Das and R. Maheshwari, "Fault Classifi- 
cation Technique for Series Compensated Transmission Line Using Support Vector Machine," International Journal of Electrical Power \& Energy Systems, Vol. 32, No. 6, July 2010, pp.629-636.

[13] P. K. Dash, S. R. Samantray and G. Panda, "Fault Classification and Section Identification of an Advanced Series-Compensated Transmission Line Using Support Vector Machine," IEEE Transactions on Power Delivery, Vol. 22, No. 1, January 2007, pp. 67-73. doi:10.1109/TPWRD. 2006.876695

[14] A. K. Pardhan, A. Routray, S. Pati and D. K. Pardhan, "Wavelet Fuzzy Combined Approach for Fault Classification of a Series-Compensated Transmission Line," IEEE Transactions on Power Delivery, Vol. 19, No. 4, October 2004, pp. 1612-1618. doi:10.1109/TPWRD.2003. 822535

[15] A. Y. Abdelaziz, A. M. Ibrahim, M. M. Mansour and H. E. Talaat, "Modern Approaches for Protection of Series-Compensated Transmission Lines," International Journal of Electric Power Systems Research, Vol. 75, No. 1, July 2005, pp. 85-98. doi:10.1016/j.epsr.2004.10.016

[16] M. El-Moursi, A. M. Sharaf and K. El-Arroudi, "Optimal Control Schemes for SSSC for Dynamic Series Compensation," International Journal of Electric Power Systems Research, Vol. 78, No. 4, April 2008, pp. 646-656. doi:10. 1016/j.epsr.2007.05.009

[17] Z. Y. He, X. Q. Chen and G. M. Luo, "Wavelet Entropy

\section{Appendix}

\section{System Parameters of Figure 2 (Base MVA = 100)}

Area 1:

Rated Voltage: $13.8 \mathrm{kV}$

Short Circuit Capacity: 21000 MVA

Area 2:

Rated Voltage: $735 \mathrm{kV}$

Short Circuit Capacity: 30000 MVA

Transformer $1(\Delta / \mathrm{Y})$ : Rated Voltage: $13.8 / 735 \mathrm{kV}$

Rated Power: 2100 MVA

Leakage Resistance: $0.002 \mathrm{pu}$

Leakage Reactance: $0.08 \mathrm{pu}$

Transformer 2 (Y/Y): Rated Voltage: 735/230 kV

Rated Power: 300 MVA

Leakage Resistance: $0.002 \mathrm{pu}$

Leakage Reactance: $0.15 \mathrm{pu}$

Transmission Lines: Resistance: $0.001 \mathrm{pu}$

Reactance: $0.0195 \mathrm{pu}$

Loads: Load 1: $100 \mathrm{MW}$

Loads 2 and 3: 1.32 MW, 330MVAR
Definition and Its Application for Transmission Line Fault Detection and Identification (Part II: Fault Detection in Transmission Line)," Proceedings of International Conference on Power System Technology, Chongqing, October 2006, pp. 1-5.

[18] S. El-Safty, and A. M. El-Zonkoly, "Applying Wavelet Entropy Principle in Fault Classification”, International Journal of Electrical Power \& Energy Systems, Vol. 31, No. 10, November-December 2009, pp. 604-607. doi:10. 1016/j.ijepes.2009.06.003

[19] Z. Y. He, X. Q. Chen and G. M. Luo, "Wavelet Entropy Definition and Its Application for Transmission Line Fault Detection and Identification (Part I: Definition and Methodology)," Proceedings of International Conference on Power System Technology, Chongqing, October 2006, pp. 1-5.

[20] Z. Y. He, X. Q. Chen and G. M. Luo, "Wavelet Entropy Definition and Its Application for Transmission Line Fault Detection and Identification (Part III: Transmission Line Faults Transients Identification)," Proceedings of International Conference on Power System Technology, Chongqing, October 2006, pp. 1-5.

[21] Z. M. Li, W. X. Li and R. Y. Liu, "Applications of Entropy Principles in Power System: A Survey," IEEE/PES Transmission and Distribution Conference and Exhibition, Dalian, August 2005, pp. 1-4.

SSSC:

\section{Load 4: 250MW}

Load 5: 300MW

Rated Power: 100 MVA

Nominal DC Voltage: $20 \mathrm{kV}$

Nominal AC Voltage: $138 \mathrm{kV}$

Number of Pulses: 48 pulse

UPFC: SSSC and STATCOM each;

Rated power: $100 \mathrm{MVA}$

Nominal DC Voltage: $20 \mathrm{kV}$

Nominal AC Voltage: $138 \mathrm{kV}$

Number of Pulses: 48 pulse

Series Coupling Transformer (Y/Y):

Rated Voltage: 138/147 kV

Rated Power: 100 MVA

Leakage Resistance: $0.002 \mathrm{pu}$

Leakage Reactance: $0.05 \mathrm{pu}$

Shunt Coupling Transformer (Y/Y):

Rated Voltage: 138/735 kV

Rated Power: 100 MVA

Leakage Resistance: $0.002 \mathrm{pu}$

Leakage Reactance: $0.02 \mathrm{pu}$ 